\title{
In Vitro Assessment of 17 Antimicrobial Agents Against Clinical Mycobacterium Avium Complex Isolates
}

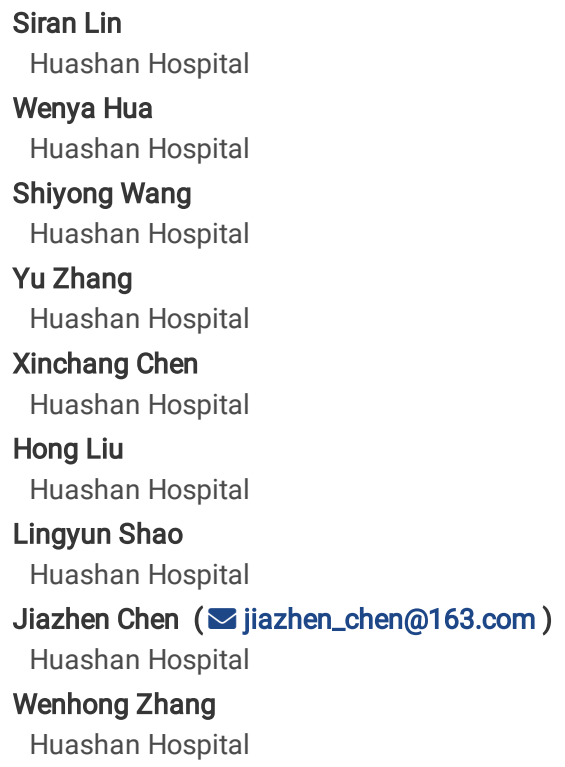

Keywords: Mycobacterium avium complex (MAC), Drug susceptibility test, Minimum inhibitory concentration (MIC), Mycobacterium intracellulare, Mycobacterium avium

Posted Date: December 22nd, 2021

DOI: https://doi.org/10.21203/rs.3.rs-1142834/v1

License: (a) (i) This work is licensed under a Creative Commons Attribution 4.0 International License. Read Full License 


\section{Abstract}

Background: Recently, Mycobacterium avium complex (MAC) infections have been increasing, especially in immunocompromised and older adults. The rapid increase has triggered a global health concern due to limited therapeutic strategies and adverse effects caused by long-term medication. To provide more evidence for the treatment of MAC, we studied the in vitro inhibitory activities of 17 antimicrobial agents against clinical MAC isolates.

Results: A total of 111 clinical MAC isolates were enrolled in the study and they were identified as M. intracellulare, M. avium, M. marseillense, M. colombiense, M. yongonense, $M$. arosience, and one isolate was undifferentiated at subspecies level. MAC strains had relatively low (0-21.6\%) resistance to clarithromycin, amikacin, bedaquiline, rifabutin, streptomycin, and clofazimine, and the resistant rates to isoniazid, rifampin, linezolid, doxycycline, and ethionamide were very high (72.1-100\%). In addition, M. avium and M. intracellulare had different resistance profiles for clarithromycin, ethambutol, trimethoprim/sulfamethoxazole, amikacin, linezolid, clofazimine, cycloserine, and ethionamide.

Conclusions: Our results supported the current usage of macrolides, rifabutin, and aminoglycosides in the regimens for MAC infection, and also demonstrated the low resistance rate against new drugs, such as clofazimine, tedizolid, and bedaquiline, suggesting the possible implementation of these drugs in MAC treatment.

\section{Background}

Members of the Mycobacterium avium complex (MAC) are the most common nontuberculous mycobacteria (NTM) species that cause pulmonary, soft tissue, and systemic diseases. MAC tends to cause infection in people with immunodeficiencies or underlying lung diseases. Host factors associated with MAC infection include acquired immunodeficiency syndrome, gene mutations in the interferon gamma (IFN- $\gamma$ )-interleukin 12 axis, positive anti-IFN- $\gamma$ autoantibodies, cystic fibrosis, and bronchiectasis[1-3]. Over the last decade, the incidence of MAC infections have increased, along with the emergence of several novel subspecies. Mycobacterium intracellulare and Mycobacterium avium remain the most important and prevalent pathogens in the MAC, while other subspecies, including Mycobacterium chimaera, Mycobacterium colombiense, and Mycobacterium marseillense in the MAC have been increasingly reported recently.

M. chimaera, one of the subspecies of $M$. intracellulare, is transmitted through contaminated catheters and often causes disseminated and life-threatening infections in people who have undergone open-heart surgery[4, 5]. As for $M$. colombiense and $M$. marseillense, they are genetically different from $M$. avium and $M$. intracellulare[6, 7]. M. colombiense was first reported in Columbian patients with human immunodeficiency virus[8], and has since been isolated from both immunocompromised and immunocompetent patients with cutaneous, lymph node, and pulmonary infections[9-11]. M. marseillense, which was identified later in 2009, has similar pathogenicity to M. colombiense. For Other subspecies, like Mycobacterium vulneris, Mycobacterium timonense, Mycobacterium arosiense, Mycobacterium yongonense, and Mycobacterium bouchedurhonense, few cases were reported.

MAC infections can be difficult to treat due to multiple factors, including environmental and genetic risk factors and frequent drug-related side effects. A culture conversion rate of $50 \%-80 \%$, a recurrence rate of $25 \%-48 \%$, and a reinfection rate of $46 \%-75 \%$ have been observed in patients with MAC lung diseases (MAC-LD)[12-14]. Treatment guidelines for MAC-LD by the American Thoracic Society and the British Thoracic Society recommended a three-drug therapeutic approach that includes macrolides, rifampin, and ethambutol[15]. Additionally, for patients with refractory, severe or macrolide-resistant MAC-LD, parenteral amikacin or streptomycin are recommended treatments. In the MAC treatment regimen, only macrolides and amikacin undergo drug susceptibility testing[1618], as the other agents lack correlations between in vitro testing and in vivo clinical response. Recently, a limited number of new antibiotics, including antituberculous agents, such as clofazimine, bedaquiline, and tedizolid, have been introduced to treat MAC.

Although in vitro drug susceptibility testing of MAC is routine, novel drugs are rarely tested. In addition, the prevalent MAC subspecies differ by regions, which could cause different resistance profiles of MAC from different regions. Therefore, we conducted subspecies identification and drug susceptibility testing on the MAC strains collected from patients admitted to our hospital in Shanghai, China. In addition to the frequently used drugs, we also tested clofazimine, bedaquiline, tedizolid, and cycloserine, with the aim of exploring the effectiveness of antimicrobials against MAC.

\section{Methods}

\section{Study design, isolate collection and species identification}

Between January 2017 and December 2020, a total of 111 MAC clinical isolates were collected from Huashan Hospital affiliated to Fudan University, Shanghai, China. The MAC isolates were cultured in the Middlebrook 7H9 media supplemented with 10\% oleic acid/dextrose/catalase (OADC). The MAC subspecies were identified by partial sequences of the $h s p 65$ and rpoB genes and a phylogenetic tree was analyzed based on these genes. The $h s p 65 \mathrm{gene}$ was amplified with primers TB11 (5'-AGTTTGATCCTGGCTCAG-3') and TB12 (5'-GGTTACCTTGTTACGACTT-3')[47] and the rpoB gene was amplified with primers MycoF (5'-CGATGCGGTAAAGGTGACATTG-3') and MycoR (5'-CCTTGACAGTGGACACCTTGGA-3')[48]. The phylogenetic tree was built using the MEGA software version 10.0 by the Neighbor joining method with a bootstrap value 1,000. The sequences of $h s p 65$ and rpoB of MAC type strains, $M$. avium subsp. avium ATCC25291, M. intracellulare ATCC13950, M. intracellulare FLAC0133, M. intracellulare FLAC0181, M. intracellulare MOTT-02, M. marseillense DSM45437, M. yongonense 05-1390, M. colombiense CECT3035, M. vulneris DSM45247, M. mantenii DSM45255, M. arosiense DSM45069, M. paraintracellulare MOTT-64, and M. intracellulare subsp. chimaera DSM44623 were used as references.

\section{Drug susceptibility testing}

The Sensititre Myco susceptibility plate for slow-growing mycobacteria (Thermo Fisher Scientific Inc., Waltham, MA, USA) was used to test the susceptibility of the following antimicrobial agents: clarithromycin, rifabutin, ethambutol, isoniazid, moxifloxacin, rifampin, trimethoprim/sulfamethoxazole, amikacin, linezolid, ciprofloxacin, streptomycin, doxycycline and ethionamide, according to the manufacturer protocol. Bedaquiline was purchased from AmBeed Inc. 
(Arlington Heights, IL, USA). Clofazimine, tedizolid and cycloserine were purchased from Aladdin (Shanghai, China). The drug susceptibility testing of bedaquiline, clofazimine, tedizolid, and cycloserine was performed using broth microdilution method according to Clinical and Laboratory Standards Institute (CLSI) protocol M24-A3. The 111 clinical MAC isolates were cultured on Middlebrook 7H11 agar for 7-14 days. M. intracellulare ATCC13950, Staphylococcus aureus ATCC29215, and Mycobacterium smegmatis ATCC19420 were used as controls. Then isolates were transferred to the Middlebrook $7 \mathrm{H} 9$ media supplemented with $10 \%$ OADC and cultured for one week at $37^{\circ} \mathrm{C}$. The bacterial suspension was adjusted to a $1 \mathrm{McF}$ arland standard with sterile demineralized water and was transferred to the Middlebrook $7 \mathrm{H} 9$ media with $10 \% \mathrm{OADC}$ at a ratio of 1:100. For tests using the Sensititre Myco susceptibility plate, $100 \mu \mathrm{L}$ of the inoculum solution was added to each well of the 96 -well microtitre plate containing lyophilized antibiotics. For the other four antimicrobial agents, $100 \mu \mathrm{L}$ of both inoculum solution and serial dilutions of the agents were added to the 96 -well plates. Plates were covered with adhesive seals and incubated at $37^{\circ} \mathrm{C}$ in ambient air for 14 days. Results were read manually by visual growth readings according to the CLSI M24 guidelines and illustrations of various growth patterns. The minimum inhibitory concentration (MIC) values were the lowest concentrations that completely inhibited growth except for trimethoprim/sulfamethoxazole, for which the MIC value was read as the lowest concentration that inhibited $80 \%$ of the growth compared to the positive control. MIC breakpoints of the antibiotics for MAC are shown in Table 1.

Table 1

Breakpoints of 17 antibiotics.

\begin{tabular}{|c|c|c|c|}
\hline \multirow[t]{2}{*}{ Antimicrobial agent } & \multicolumn{3}{|c|}{ MIC breakpoints $(\mu \mathrm{g} / \mathrm{mL})$} \\
\hline & Susceptibility & Intermediate & Resistance \\
\hline $\mathrm{CLA}^{\mathrm{a}}$ & $\leq 8$ & 16 & $\geq 32$ \\
\hline $\mathrm{RFB}^{\mathrm{b}}$ & $\leq 2$ & - & $\geq 4$ \\
\hline $\mathrm{EMB}^{\mathrm{c}}$ & - & - & $>5$ \\
\hline $\mathrm{INH}^{\mathrm{C}}$ & - & - & $>0.2$ \\
\hline MXFa & $\leq 1$ & 2 & $\geq 4$ \\
\hline $\mathrm{RIF}^{\mathrm{b}}$ & $\leq 1$ & - & $\geq 2$ \\
\hline $\mathrm{SXT}^{\mathrm{b}}$ & $\leq 2 / 38$ & - & $\geq 4 / 76$ \\
\hline $\mathrm{AMI}^{\mathrm{a}}$ & $\leq 16$ & 32 & $\geq 64$ \\
\hline LZD $^{a}$ & $\leq 8$ & 16 & $\geq 32$ \\
\hline$C P^{b}$ & $\leq 1$ & 2 & $\geq 4$ \\
\hline $\mathrm{STR}^{\mathrm{d}}$ & $\leq 16$ & 32 & $\geq 64$ \\
\hline$D O X^{b}$ & $\leq 1$ & $2-4$ & $\geq 8$ \\
\hline $\mathrm{ETH}^{\mathrm{c}}$ & - & - & $>5$ \\
\hline $\mathrm{TZD}^{\mathrm{e}}$ & - & - & $>8$ \\
\hline $\mathrm{CFZ}^{\mathrm{d}}$ & $\leq 1$ & 2 & $\geq 4$ \\
\hline $\mathrm{BDQ}^{f}$ & - & - & $>0.25$ \\
\hline $\mathrm{CS}^{\mathrm{d}}$ & $\leq 16$ & 32 & $\geq 64$ \\
\hline
\end{tabular}

\section{Results}

A total of 111 MAC isolates were collected and were identified as $M$. intracellulare $(\mathrm{n}=81), M$. avium $(\mathrm{n}=13), M$. marseillense $(\mathrm{n}=7), M$. colombiense $(\mathrm{n}=7)$, $M$. yongonense $(\mathrm{n}=1)$, and $M$. arosience $(\mathrm{n}=1)$ (Fig. 1). One isolate, named 18-T1838, was similar to both M. vulneris and M. colombiense in the phylogenetic tree and could not be identified to the subspecies level.

We tested the antimicrobial activities of 17 antimicrobial agents against $111 \mathrm{MAC}$ isolates (Table 2). The detailed MIC values of different subspecies were listed in Table S1. The MAC isolates showed a low resistance rate to commonly used drugs, such as clarithromycin (4.5\%), amikacin (2.7\%), rifabutin (21.6\%), and streptomycin (17.1\%). However, they were highly resistant to most anti-tuberculosis drugs, such as isoniazid (100\%), rifampin (82.9\%), linezolid (72.1\%), doxycycline (98.2\%), and ethionamide (91.9\%). Specifically, all the MAC isolates were resistant to isoniazid. Besides, ciprofloxacin also showed a poor inhibitory effect on MAC isolates which had a resistance rate of $87.4 \%$. Furthermore, the MAC isolates showed an intermediate resistance rate (54.1\%, $62.2 \%$, and $60.4 \%$ ) for ethambutol, trimethoprim/sulfamethoxazole, and moxifloxacin, respectively. Interestingly, the MAC isolates showed a low resistance rate for all four newly used drugs: bedaquiline (0\%), clofazimine (19.8\%), tedizolid $(26.1 \%)$, and cycloserine $(30.6 \%)$. 
Table 2

Drug resistant rates of different MAC subspecies.

\begin{tabular}{|c|c|c|c|c|c|c|c|c|}
\hline \multirow[t]{3}{*}{ Antimicrobial agent } & \multicolumn{8}{|c|}{ No. of resistant isolates (\%) } \\
\hline & All isolates & M. intracellulare & M. avium & M. marseillense & M. colombiense & M. yongonense & M. arosience & 18-T1838 \\
\hline & $n=111$ & $\mathrm{n}=\mathbf{8 1}$ & $n=13$ & $\mathrm{n}=7$ & $n=7$ & $n=1$ & $\mathrm{n}=1$ & $n=1$ \\
\hline CLA & $5(4.5 \%)$ & $3(3.7 \%)$ & $2(15.4 \%)$ & $0(0 \%)$ & $0(0 \%)$ & 0 & 0 & 0 \\
\hline RFB & $24(21.6 \%)$ & $17(21.0 \%)$ & $2(15.4 \%)$ & $1(14.3 \%)$ & $3(42.9 \%)$ & 0 & 1 & 0 \\
\hline EMB & $60(54.1 \%)$ & $33(40.7 \%)$ & $12(92.3 \%)$ & $6(85.7 \%)$ & $7(100 \%)$ & 1 & 1 & 0 \\
\hline $\mathrm{INH}$ & $111(100 \%)$ & $81(100 \%)$ & $13(100 \%)$ & $7(100 \%)$ & $7(100 \%)$ & 1 & 1 & 1 \\
\hline MXF & $67(60.4 \%)$ & $43(53.1 \%)$ & $8(61.5 \%)$ & $6(85.7 \%)$ & $7(100 \%)$ & 1 & 1 & 1 \\
\hline RIF & $92(82.9 \%)$ & $64(79.0 \%)$ & $11(84.6 \%)$ & $7(100 \%)$ & $7(100 \%)$ & 1 & 1 & 1 \\
\hline SXT & $69(62.2 \%)$ & $45(55.6 \%)$ & $10(76.9 \%)$ & $6(85.7 \%)$ & $5(71.4 \%)$ & 1 & 1 & 1 \\
\hline AMI & $3(2.7 \%)$ & $1(1.2 \%)$ & $2(15.4 \%)$ & $0(0 \%)$ & $0(0 \%)$ & 0 & 0 & 0 \\
\hline LZD & $80(72.1 \%)$ & $53(65.4 \%)$ & $11(84.6 \%)$ & $7(100 \%)$ & $6(85.7 \%)$ & 1 & 1 & 1 \\
\hline CIP & $97(87.4 \%)$ & $70(86.4 \%)$ & $10(76.9 \%)$ & $7(100 \%)$ & $7(100 \%)$ & 1 & 1 & 1 \\
\hline STR & $19(17.1 \%)$ & $17(21.0 \%)$ & $2(15.4 \%)$ & $0(0 \%)$ & $0(0 \%)$ & 0 & 0 & 0 \\
\hline DOX & $109(98.2 \%)$ & $79(97.5 \%)$ & $13(100 \%)$ & $7(100 \%)$ & $7(100 \%)$ & 1 & 1 & 1 \\
\hline ETH & $102(91.9 \%)$ & $75(92.6 \%)$ & $10(76.9 \%)$ & $7(100 \%)$ & $7(100 \%)$ & 1 & 1 & 1 \\
\hline TZD & $29(26.1 \%)$ & $24(29.6 \%)$ & $4(30.8 \%)$ & $0(0 \%)$ & $1(14.3 \%)$ & 0 & 0 & 0 \\
\hline CFZ & $22(19.8 \%)$ & $14(17.3 \%)$ & $4(30.8 \%)$ & $2(28.6 \%)$ & $2(28.6 \%)$ & 0 & 0 & 0 \\
\hline $\mathrm{BDQ}$ & $0(0 \%)$ & $0(0 \%)$ & $0(0 \%)$ & $0(0 \%)$ & $0(0 \%)$ & 0 & 0 & 0 \\
\hline $\mathrm{CS}$ & $34(30.6 \%)$ & $21(25.9 \%)$ & $9(69.2 \%)$ & $1(14.3 \%)$ & $2(28.6 \%)$ & 1 & 0 & 0 \\
\hline
\end{tabular}

Most agents showed similar antimicrobial activities against the two main MAC subspecies, $M$. intracellulare and $M$. avium. However, $M$. avium had a higher resistance rate than that of $M$. intracellulare for clarithromycin ( $3.7 \%$ vs $15.4 \%)$, ethambutol ( $40.7 \%$ vs $92.3 \%)$, trimethoprim/sulfamethoxazole ( $55.6 \%$ vs $76.9 \%$ ), amikacin (1.2\% vs $15.4 \%$ ), linezolid (65.4\% vs $84.6 \%$ ), clofazimine (17.3\% vs $30.8 \%)$, and cycloserine ( $25.9 \%$ vs $69.2 \%)$. M. intracellulare had a higher resistance to ethionamide than $M$. avium. All or almost all the $M$. marseillense and $M$. colombiense isolates were resistant to ethambutol, isoniazid, moxifloxacin, rifampin, trimethoprim/sulfamethoxazole, linezolid, ciprofloxacin, doxycycline, and ethionamide, while none of them were resistant to clarithromycin, amikacin, streptomycin, or bedaquiline (Table 2). The other agents showed good inhibitory activities against the two subspecies which had a resistance rate ranging from $0-42.9 \%$. M. yongonense, $M$. arosience, and 18-T1838 isolates had similar resistance profiles against the 17 antimicrobial agents, except for that $M$. yongonense was resistant to cycloserine ( $\mathrm{MIC}>64 \mu \mathrm{g} / \mathrm{mL})$ and $M$. arosience was resistant to rifabutin.

\section{Discussion}

Our antibiotic susceptibility testing results supported the current recommendation of using macrolides, rifamycins, and aminoglycosides to treat MAC infections. In our study, clarithromycin showed good inhibitory activity against all MAC isolates, consistent with previous studies[19, 20]. The resistance rate of MAC isolates against rifampin was 82.9\% (92/111), which was in agreement (78.9\%; 216/274) with a previous study[21]. Unlike rifampin, rifabutin showed a better antimicrobial activity and was recommended as an alternative to rifampicin, especially for disseminated MAC infections, for patients infected with MAC[22]. However, in a recent study, neither rifampin or rifabutin inhibited MAC growth in vitro[23]. Therefore, further clinical trials are still needed to determine the best choice among different rifamycins for treating MAC diseases. The intermediate resistance against ethambutol was comparable with that of a previous study (58.1\%;159/274)[21]. These results do not support the usage of ethambutol for MAC. Among the aminoglycosides, amikacin may be better for treating MAC infections than streptomycin, with an overall low resistant rate of $2.7 \%$ (24/111), which is as low as shown in previous studies[24, 25]. Streptomycin is a potentially good choice for treatment of MAC isolates. In a study in a Taiwanese district, the resistance rate of MAC isolates against streptomycin was even lower $(4.8 \% ; 4 / 83)[26]$. This difference may be regional (different geographies) or may be due to the inconsistent proportions of MAC subspecies collected in the studies.

As second-line drugs for MAC disease, the clinical efficacy of moxifloxacin and linezolid remains uncertain[27]. In our study, both had limited activity against MAC isolates, which is comparable with previous studies in Korea[28], Sweden[25], and China[29]. However, unlike the poor activity in vitro, a recent study has shown that fluoroquinolone-containing regimens could achieve similar clinical improvement with the standard regimen and could be an alternative for patients who cannot tolerate the standard regimen[30]. As for the other tested anti-tuberculosis drugs, such as isoniazid, ciprofloxacin, doxycycline, and ethionamide, the MAC isolates showed high resistance, which supported the consensus that these drugs should not be used in the treatment of MAC diseases as shown in a previous study[20]. The comparison of drug resistance rate of recommended agents for MAC isolates from different studies were shown in Table 3. 
Table 3

The comparison of drug resistance rate of recommended agents for MAC isolates from different studies.

\begin{tabular}{|c|c|c|c|c|c|c|c|c|c|c|}
\hline Nation/district & Year & Isolate & CLA & RIF & EMB & MXF & RFB & AMI & LZD & STR \\
\hline This study & 2021 & 111 & $5(4.5 \%)$ & $92(82.9 \%)$ & $60(54.1 \%)$ & $67(60.4 \%)$ & $24(21.6 \%)$ & $3(2.7 \%)$ & $80(72.1 \%)$ & $19(17.1 \%)$ \\
\hline Germany & 2020 & 98 & $1(1.2 \%)$ & - & - & $38(44.7 \%)$ & - & $0(0 \%)$ & $57(67.1 \%)$ & - \\
\hline Germany & 2019 & 683 & $17(2.5 \%)$ & - & - & $430(63.1 \%)$ & - & - & $511(75.0 \%)$ & - \\
\hline Korea & 2018 & 1883 & $95(5.0 \%)$ & $1080(57.4 \%)$ & 1691(89.8\%) & $1054(56.0 \%)$ & - & $166(8.8 \%)$ & $805(42.8 \%)$ & - \\
\hline Sweden & 2017 & 229 & $6(2.6 \%)$ & $210(91.7 \%)$ & - & $112(48.9 \%)$ & - & $11(4.8 \%)$ & $118(51.5 \%)$ & - \\
\hline Taiwan & 2018 & 83 & $0(0 \%)$ & - & - & $72(86.7 \%)$ & - & $2(2.4 \%)$ & $61(73.5 \%)$ & $4(4.8 \%)$ \\
\hline UK & 2016 & - & $248(19.9 \%)$ & $686(55.7 \%)$ & $391(31.9 \%)$ & - & $58(5.9 \%)$ & $100(8.2 \%)$ & - & $498(53.0 \%)$ \\
\hline
\end{tabular}

In our study, the new oxazolidinone, tedizolid, had a significantly lower resistance rate than linezolid, supporting the previous results which indicated that tedizolid has enhanced in vitro activities against several NTM species[31]. In addition, it has less side effects in long-term therapy, compared with linezolid and has a concentration-dependent activity against $M$. avium. Its efficacy can be enhanced by ethambutol, which suggests its potential role in the treatment of MAC diseases[32].

Clofazimine, which also had a low resistance rate in our study, has been recently proven to be an effective agent for the treatment of MAC both in patients and mouse models[33,34]. A recent study conducted in Korea found that a lower MIC value of clofazimine $(\leq 0.25 \mathrm{mg} / \mathrm{L})$ was associated with negative conversion of sputum culture in patients with NTM lung diseases[35]. Another study in Korea demonstrated that clofazimine, together with inhaled amikacin, could provide favorable outcomes in patients with refractory MAC-LD[36]. Nevertheless, the adverse effects of clofazimine are a major concern that affects its application in patients.

Bedaquiline is a diarylquinoline antibiotic, acting through an antimicrobial mechanism by inhibiting F1Fo-ATP synthase, an enzyme that is essential in Mycobacterium tuberculosis[36]. Although several clinical studies have found increased sputum conversion rates with bedaquiline in patients with multidrugresistant tuberculosis, its efficacy in the treatment of MAC-LD is currently controversial. In some studies, bedaquiline is considered to be a good candidate for refractory or relapsing diseases caused by MAC[38,39], while in other studies, bedaquiline treatment in patients with MAC-LD were not favorable due to the emergence of resistance and the decreased systemic exposure caused by rifamycin through the induction of cytochrome P450[40, 41]. In our study, most MAC isolates showed low MIC values $(0.015-0.12 \mu \mathrm{g} / \mathrm{mL})$ for bedaquiline, which is in agreement with previous studies[42-44]. Clinical trials are warranted to correlate the in vitro susceptibility of MAC to bedaquiline with the clinical outcome.

Cycloserine is mainly used to treat drug-resistant M. tuberculosis, and there are few reports on its effect on NTM. MAC isolates were completely sensitive to cycloserine in several studies[45], with an MIC breakpoint of $80 \mu \mathrm{g} / \mathrm{mL}$. However, in our study, the resistant rates ( $\geq 64 \mu \mathrm{g} / \mathrm{mL})$ are $28.9 \%$ and $42.9 \%$ for $M$. intracellulare and $M$. avium, respectively. Considering the side effects of long-term use of cycloserine and the intermediate resistance rate in vitro, it is necessary to be cautious and more data are needed to test its effect upon clinical application as a candidate drug.

In this study, drug susceptibilities of $M$. avium and $M$. intracellulare (which has been less reported) to several agents were different. M. avium had a higher resistance rate than $M$. intracel/ulare for clarithromycin, ethambutol, trimethoprim/sulfamethoxazole, amikacin, linezolid, clofazimine, and cycloserine. However, since the number of resistant M. avium isolates was small in our study, the calculations of the resistance rates of clarithromycin and amikacin using only two isolates might be inaccurate. In another study in Germany[20], higher resistance rates of $M$. avium to trimethoprim/sulfamethoxazole and linezolid were also reported. Contrary to our results, in a recent study in Shanghai[46], $M$. intracellulare had higher resistance rates than $M$. avium for most tested antimicrobials. The difference may be related to different identification methods between these studies. As for the other subspecies, few studies have reported their resistance profiles and more research is needed in the future.

\section{Conclusions}

In conclusion, clarithromycin, rifabutin, amikacin, and streptomycin showed good in vitro antimicrobial activities against the MAC isolates, with resistance rates of less than $25 \%$. However, isoniazid, rifampin, linezolid, doxycycline, and ethionamide had poor inhibitory activities, which is consistent with previous studies, and thus, not suitable to treat MAC diseases. In addition, new drugs, such as clofazimine, tedizolid, bedaquiline, and cycloserine also showed good antimicrobial activities in vitro and could be introduced to treat MAC in the future. Besides, different resistance profiles for several drugs were seen for $M$. avium and $M$. intracellulare, but further studies are still needed to confirm these differences.

\section{Abbreviations}

CLSI: Clinical and Laboratory Standards Institute; IFN-y: interferon gamma; MAC: Mycobacterium avium complex; MAC-LD: Mycobacterium avium complex lung diseases; NTM: nontuberculous mycobacteria; OADC: oleic acid/dextrose/catalase; CLA, clarithromycin; RFB, rifabutin; EMB, ethambutol; INH, isoniazid; MXF, moxifloxacin; RIF, rifampin; SXT, trimethoprim/sulfamethoxazole; AMI, amikacin; LZD, linezolid; CIP, ciprofloxacin; STR, streptomycin; DOX, doxycycline; $\mathrm{ETH}$, ethionamide; TZD, tedizolid; $\mathrm{CFZ}$, clofazimine; $\mathrm{BDQ}$, bedaquiline; $\mathrm{CS}$, cycloserine. 


\section{Declarations}

\section{Ethics approval and consent to participate}

Not applicable.

\section{Consent for publication}

Not applicable.

\section{Availability of data and materials}

All data generated or analyzed during this study are included in this article and its supplementary information files.

\section{Competing interests}

The authors declare no conflict of interest.

\section{Funding}

This work was supported by the Key Laboratory Project of Shanghai Science and Technology Commission [grant numbers 20dz2210400]

\section{Authors' contributions}

JC, LS, and WZ designed the study. SL, WH, SW, YZ, and XC performed the experimental work. SW and HL collected the data. SL, WH, and JC analyzed the data and wrote the manuscript. All authors contributed to the article and approved the submitted version.

\section{Acknowledgments}

The grant received from the Key Laboratory Project of Shanghai Science and Technology Commission is thankfully acknowledged.

\section{References}

1. Wu UI, Holland SM: Host susceptibility to non-tuberculous mycobacterial infections. LANCET INFECT DIS 2015, 15(8):968-980.

2. Lake MA, Ambrose LR, Lipman MC, Lowe DM: "Why me, why now?" Using clinical immunology and epidemiology to explain who gets nontuberculous mycobacterial infection. BMC MED 2016, 14:54.

3. Valour F, Perpoint T, Sénéchal A, Kong XF, Bustamante J, Ferry T, Chidiac C, Ader F: Interferon-y Autoantibodies as Predisposing Factor for Nontuberculous Mycobacterial Infection. EMERG INFECT DIS 2016, 22(6):1124-1126.

4. Kimsis J, Pole I, Norvaisa I, Dumpis U, Ranka R: Characterization of Mycobacterium chimaera in a heater-cooler unit in Latvia. Infect Control Hosp Epidemiol 2020:1-3.

5. Lamagni TL, Charlett A, Phin N, Zambon M, Chand M: Invasive Mycobacterium chimaera Infections and Heater-Cooler Devices in Cardiac Surgery. EMERG INFECT DIS 2020, 26(3):632.

6. González-Pérez M, Murcia MI, Landsman D, Jordan IK, Mariño-Ramírez L: Genome sequence of the Mycobacterium colombiense type strain, CECT 3035. J BACTERIOL 2011, 193(20):5866-5867.

7. Ben SI, Cayrou C, Raoult D, Drancourt M: Mycobacterium marseillense sp. nov., Mycobacterium timonense sp. nov. and Mycobacterium bouchedurhonense sp. nov., members of the Mycobacterium avium complex. Int J Syst Evol Microbiol 2009, 59(Pt 11):2803-2808.

8. Murcia MI, Tortoli E, Menendez MC, Palenque E, Garcia MJ: Mycobacterium colombiense sp. nov., a novel member of the Mycobacterium avium complex and description of MAC-X as a new ITS genetic variant. Int J Syst Evol Microbiol 2006, 56(Pt 9):2049-2054.

9. Poulin S, Corbeil C, Nguyen M, St-Denis A, Côté L, Le Deist F, Carignan A: Fatal Mycobacterium colombiense/cytomegalovirus coinfection associated with acquired immunodeficiency due to autoantibodies against interferon gamma: a case report. BMC INFECT DIS 2013, 13:24.

10. Gao W, Chen H, Jiang H, Wang Q, Tang M, Wang HS: Disseminated cutaneous infection caused by Mycobacterium colombiense. Acta Derm Venereol 2014, 94(6):727-728.

11. Esparcia O, Navarro F, Quer M, Coll P: Lymphadenopathy caused by Mycobacterium colombiense. J CLIN MICROBIOL 2008, 46(5):1885-1887.

12. Koh WJ, Moon SM, Kim SY, Woo MA, Kim S, Jhun BW, Park HY, Jeon K, Huh HJ, Ki CS et al: Outcomes of Mycobacterium avium complex lung disease based on clinical phenotype. EUR RESPIR J 2017, 50(3).

13. Wallace RJ, Brown-Elliott BA, McNulty S, Philley JV, Killingley J, Wilson RW, York DS, Shepherd S, Griffith DE: Macrolide/Azalide therapy for nodular/bronchiectatic mycobacterium avium complex lung disease. CHEST 2014, 146(2):276-282. 
14. Boyle DP, Zembower TR, Qi C: Relapse versus Reinfection of Mycobacterium avium Complex Pulmonary Disease. Patient Characteristics and Macrolide Susceptibility. Ann Am Thorac Soc 2016, 13(11):1956-1961.

15. Daley CL, laccarino JM, Lange C, Cambau E, Wallace RJ, Andrejak C, Böttger EC, Brozek J, Griffith DE, Guglielmetti L et al: Treatment of nontuberculous mycobacterial pulmonary disease: an official ATS/ERS/ESCMID/IDSA clinical practice guideline. EUR RESPIR J 2020, 56(1).

16. Griffith DE, Brown-Elliott BA, Langsjoen B, Zhang Y, Pan X, Girard W, Nelson K, Caccitolo J, Alvarez J, Shepherd S et al: Clinical and molecular analysis of macrolide resistance in Mycobacterium avium complex lung disease. Am J Respir Crit Care Med 2006, 174(8):928-934.

17. Olivier KN, Griffith DE, Eagle G, McGinnis JN, Micioni L, Liu K, Daley CL, Winthrop KL, Ruoss S, Addrizzo-Harris DJ et al: Randomized Trial of Liposomal Amikacin for Inhalation in Nontuberculous Mycobacterial Lung Disease. Am J Respir Crit Care Med 2017, 195(6):814-823.

18. Griffith DE, Eagle G, Thomson R, Aksamit TR, Hasegawa N, Morimoto K, Addrizzo-Harris DJ, O'Donnell AE, Marras TK, Flume PA et al: Amikacin Liposome Inhalation Suspension for Treatment-Refractory Lung Disease Caused by Mycobacterium avium Complex (CONVERT). A Prospective, Open-Label, Randomized Study. Am J Respir Crit Care Med 2018, 198(12):1559-1569.

19. Wetzstein N, Kohl TA, Andres S, Schultze TG, Geil A, Kim E, Biciusca T, Hügel C, Hogardt M, Lehn A et al: Comparative analysis of phenotypic and genotypic antibiotic susceptibility patterns in Mycobacterium avium complex. INT J INFECT DIS 2020, 93:320-328.

20. Maurer FP, Pohle P, Kernbach M, Sievert D, Hillemann D, Rupp J, Hombach M, Kranzer K: Differential drug susceptibility patterns of Mycobacterium chimaera and other members of the Mycobacterium avium-intracellulare complex. Clin Microbiol Infect 2019, 25(3):371-379.

21. Kwon BS, Kim MN, Sung H, Koh Y, Kim WS, Song JW, Oh YM, Lee SD, Lee SW, Lee JS et al: In Vitro MIC Values of Rifampin and Ethambutol and Treatment Outcome in Mycobacterium avium Complex Lung Disease. Antimicrob Agents Chemother 2018, 62(10).

22. Shafran SD, Singer J, Zarowny DP, Phillips P, Salit I, Walmsley SL, Fong IW, Gill MJ, Rachlis AR, Lalonde RG et al: A comparison of two regimens for the treatment of Mycobacterium avium complex bacteremia in AIDS: rifabutin, ethambutol, and clarithromycin versus rifampin, ethambutol, clofazimine, and ciprofloxacin. Canadian HIV Trials Network Protocol 010 Study Group. N Engl J Med 1996, 335(6):377-383.

23. Boorgula GD, Jakkula L, Gumbo T, Jung B, Srivastava S: Comparison of Rifamycins for Efficacy Against Mycobacterium avium Complex and Resistance Emergence in the Hollow Fiber Model System. FRONT PHARMACOL 2021, 12:645264.

24. Brown-Elliott BA, lakhiaeva E, Griffith DE, Woods GL, Stout JE, Wolfe CR, Turenne CY, Wallace RJ: In vitro activity of amikacin against isolates of Mycobacterium avium complex with proposed MIC breakpoints and finding of a 16S rRNA gene mutation in treated isolates. J CLIN MICROBIOL 2013, 51(10):3389-3394.

25. Schön T, Chryssanthou E: Minimum inhibitory concentration distributions for Mycobacterium avium complex-towards evidence-based susceptibility breakpoints. INT J INFECT DIS 2017, 55:122-124.

26. Huang CC, Wu MF, Chen HC, Huang WC: In vitro activity of aminoglycosides, clofazimine, d-cycloserine and dapsone against 83 Mycobacterium avium complex clinical isolates. J Microbiol Immunol Infect 2018, 51(5):636-643.

27. Brown-Elliott BA, Woods GL: Antimycobacterial Susceptibility Testing of Nontuberculous Mycobacteria. J CLIN MICROBIOL 2019, 57(10).

28. Cho EH, Huh HJ, Song DJ, Moon SM, Lee SH, Shin SY, Kim CK, Ki CS, Koh WJ, Lee NY: Differences in drug susceptibility pattern between Mycobacterium avium and Mycobacterium intracellulare isolated in respiratory specimens. J INFECT CHEMOTHER 2018, 24(4):315-318.

29. Liu CF, Song YM, He WC, Liu DX, He P, Bao JJ, Wang XY, Li YM, Zhao YL: Nontuberculous mycobacteria in China: incidence and antimicrobial resistance spectrum from a nationwide survey. INFECT DIS POVERTY 2021, 10(1):59.

30. Shuto H, Komiya K, Goto A, Kan T, Honjo K, Uchida S, Takikawa S, Yoshimatsu T, Yamasue M, Hiramatsu K et al: Efficacy and safety of fluoroquinolonecontaining regimens in treating pulmonary Mycobacterium avium complex disease: A propensity score analysis. PLOS ONE 2020, 15 (7):e235797.

31. Yuste JR, Bertó J, Del PJ, Leiva J: Prolonged use of tedizolid in a pulmonary non-tuberculous mycobacterial infection after linezolid-induced toxicity. $J$ Antimicrob Chemother 2017, 72(2):625-628.

32. Ruth MM, Koeken V, Pennings LJ, Svensson EM, Wertheim H, Hoefsloot W, van Ingen J: Is there a role for tedizolid in the treatment of non-tuberculous mycobacterial disease? J Antimicrob Chemother 2020, 75(3):609-617.

33. Martiniano SL, Wagner BD, Levin A, Nick JA, Sagel SD, Daley CL: Safety and Effectiveness of Clofazimine for Primary and Refractory Nontuberculous Mycobacterial Infection. CHEST 2017, 152(4):800-809.

34. Lee JM, Park J, Choi S, Jhun BW, Kim SY, Jo KW, Hong JJ, Kim LH, Shin SJ: A Clofazimine-Containing Regimen Confers Improved Treatment Outcomes in Macrophages and in a Murine Model of Chronic Progressive Pulmonary Infection Caused by the Mycobacterium avium Complex. FRONT MICROBIOL 2020, 11:626216.

35. Kwak N, Whang J, Yang JS, Kim TS, Kim SA, Yim JJ: Minimal Inhibitory Concentration of Clofazimine Among Clinical Isolates of Nontuberculous Mycobacteria and Its Impact on Treatment Outcome. CHEST 2021, 159(2):517-523.

36. Kim BG, Kim H, Kwon OJ, Huh HJ, Lee NY, Baek SY, Sohn I, Jhun BW: Outcomes of Inhaled Amikacin and Clofazimine-Containing Regimens for Treatment of Refractory Mycobacterium avium Complex Pulmonary Disease. J CLIN MED 2020, 9(9).

37. Matteelli A, Carvalho AC, Dooley KE, Kritski A: TMC207: the first compound of a new class of potent anti-tuberculosis drugs. FUTURE MICROBIOL 2010, 5(6):849-858.

38. Philley JV, Wallace RJ, Benwill JL, Taskar V, Brown-Elliott BA, Thakkar F, Aksamit TR, Griffith DE: Preliminary Results of Bedaquiline as Salvage Therapy for Patients With Nontuberculous Mycobacterial Lung Disease. CHEST 2015, 148(2):499-506.

39. Vesenbeckh S, Schönfeld N, Krieger D, Bettermann G, Bauer TT, Rüssmann H, Mauch H: Bedaquiline as a potential agent in the treatment of M. intracellulare and M. avium infections. EUR RESPIR J 2017, 49(3). 
40. Alexander DC, Vasireddy R, Vasireddy S, Philley JV, Brown-Elliott BA, Perry BJ, Griffith DE, Benwill JL, Cameron AD, Wallace RJ: Emergence of mmpT5 Variants during Bedaquiline Treatment of Mycobacterium intracellulare Lung Disease. J CLIN MICROBIOL 2017, 55(2):574-584.

41. Zweijpfenning S, Schildkraut JA, Coolen J, Ruesen C, Koenraad E, Janssen A, Ruth MM, de Jong AS, Kuipers S, Aarnoutse RE et al: Failure with acquired resistance of an optimised bedaquiline-based treatment regimen for pulmonary Mycobacterium avium complex disease. EUR RESPIR J 2019, 54(1).

42. Brown-Elliott BA, Philley JV, Griffith DE, Thakkar F, Wallace RJ: In Vitro Susceptibility Testing of Bedaquiline against Mycobacterium avium Complex. Antimicrob Agents Chemother 2017, 61(2).

43. Pang Y, Zheng H, Tan Y, Song Y, Zhao Y: In Vitro Activity of Bedaquiline against Nontuberculous Mycobacteria in China. Antimicrob Agents Chemother 2017, 61(5).

44. Kim DH, Jhun BW, Moon SM, Kim SY, Jeon K, Kwon OJ, Huh HJ, Lee NY, Shin SJ, Daley CL et al: In Vitro Activity of Bedaquiline and Delamanid against Nontuberculous Mycobacteria, Including Macrolide-Resistant Clinical Isolates. Antimicrob Agents Chemother 2019, 63(8).

45. Cowman S, Burns K, Benson S, Wilson R, Loebinger MR: The antimicrobial susceptibility of non-tuberculous mycobacteria. J Infect 2016, 72(3):324-331.

46. Wang W, Yang J, Wu X, Wan B, Wang H, Yu F, Guo Y: Difference in drug susceptibility distribution and clinical characteristics between Mycobacterium avium and Mycobacterium intracellulare lung diseases in Shanghai, China. J MED MICROBIOL 2021, 70(5).

47. Telenti A, Marchesi F, Balz M, Bally F, Böttger EC, Bodmer T: Rapid identification of mycobacteria to the species level by polymerase chain reaction and restriction enzyme analysis. J CLIN MICROBIOL 1993, 31(2):175-178.

48. Ben SI, Adékambi T, Raoult D, Drancourt M: rpoB sequence-based identification of Mycobacterium avium complex species. Microbiology (Reading) 2008, 154(Pt 12):3715-3723.

49. Nguyen T, Anthony RM, Bañuls AL, Nguyen T, Vu DH, Alffenaar JC: Bedaquiline Resistance: Its Emergence, Mechanism, and Prevention. CLIN INFECT DIS 2018, 66(10):1625-1630.

\section{Figures}

Figure 1

Phylogenetic analyses of concatenated $h s p 65$ and $r p o B$ gene sequences of clinical MAC isolates, using the neighbor-joining method by MEGA10 software. The scale bar indicated the number of substitutions per nucleotide position. The type strains were indicated in each cluster, if included.

\section{Supplementary Files}

This is a list of supplementary files associated with this preprint. Click to download.

- Additionalfile1.xlsx 